\title{
A large-scale functional approach to uncover human genes and pathways in Drosophila
}

Rong Xu ${ }^{1}$, Kejing Deng ${ }^{1}$, Yi Zhu ${ }^{1}$, Yue $\mathrm{Wu}^{1}{ }^{1}$, Jing Ren ${ }^{1}$, Min Wan ${ }^{2}$, Shouyuan Zhao ${ }^{1}$, Xiaohui Wu ${ }^{1}$, Min Han ${ }^{1,2}$, Yuan Zhuang ${ }^{1,3}$, Tian $\mathrm{Xu}^{1,4}$

${ }^{1}$ Institute of Developmental Biology and Molecular Medicine and School of Life Science, Fudan University, Shanghai 200433, China; ${ }^{2}$ Howard Hughes Medical Institute and Department of MCDB, University of Colorado, Boulder, CO 80309-0347, USA; ${ }^{3}$ Department of Immunology, Duke University Medical Center, Durham, NC 27708, USA; ${ }^{4}$ Howard Hughes Medical Institute and Department of Genetics, Yale University School of Medicine, 295 Congress Avenue, BCMM236, New Haven, CT 06536, USA

We demonstrate the feasibility of performing a systematic screen for human gene functions in Drosophila by assaying for their ability to induce overexpression phenotypes. Over 1500 transgenic fly lines corresponding to 236 human genes have been established. In all, 51 lines are capable of eliciting a phenotype suggesting that the human genes are functional. These heterologous genes are functionally relevant as we have found a similar mutant phenotype caused either by a dominant negative mutant form of the human ribosomal protein L8 gene or by RNAi downregulation of the Drosophila RPL8. Significantly, the Drosophila RPL8 mutant can be rescued by wild-type human $R P L 8$. We also provide genetic evidence that Drosophila RPL8 is a new member of the insulin signaling pathway. In summary, the functions of many human genes appear to be highly conserved, and the ability to identify them in Drosophila represents a powerful genetic tool for large-scale analysis of human transcripts in vivo.

Keywords: Drosophila, human gene, GAL4/UAS, genetic screen, RPL8, insulin signaling

Cell Research (2008) 18:1114-1127. doi: 10.1038/cr.2008.295; published online 28 October 2008

\section{Introduction}

To date the genomes of 94 eukaryotic organisms have been completely sequenced (www.genomesonline.org; [1]). The major challenge is to uncover the function of the annotated and predicted genes in the various organisms. In this post-genome era, the fruit fly, mouse, and other model organisms will serve a major role as model systems for functional genome analysis.

The entire sequence of the human genome was first completed in 2001. Recent estimations indicate that the human genome may encode approximately 30000 genes. However, roughly more than $40 \%$ of all potential human open reading frames have not yet been assigned a function $[2,3]$. A complete genome sequence of an organism

Correspondence: Kejing Deng ${ }^{\mathrm{a}}$, Tian $\mathrm{Xu}{ }^{\mathrm{b}}$

${ }^{\mathrm{a}}$ E-mail: dengkj@fudan.edu.cn

${ }^{\mathrm{b}}$ Tel: +1-203-737-2623; Fax: +1-203-737-1762

E-mail: tian.xu@yale.edu

Received 13 June 2008; accepted 16 June 2008; published online 28 October 2008 can be considered to be the ultimate genetic map, since the sequence and the position of every gene along each chromosome are known. However, knowledge of the complete DNA sequence alone does not tell us directly how this genetic information is elaborated to observable phenotypes such as physical traits and behaviors. Consequently, the challenge and focus of the next phase of the Human Genome Project will be to decipher all of the functional units in a genome sequence and determine how this information will impact on our health and society [4].

Drosophila has been proven to be a powerful genetic system for identifying genes and defining signaling pathways that are involved in development. For example, studies of the wingless, hedgehog, decapentaplegic, and Notch signaling pathways in the fly have provided important insights and also helped to define the corresponding pathways in mammals. Not only are the components of these pathways evolutionarily conserved between fly and human, but they also carry out similar if not identical functions [5].

In Drosophila, an overexpression phenotype often can provide insightful information into the function of a 
gene. For example, altering either the amount or quality of a gene product in various tissues during development may shed light on whether the gene is able to act on proliferative events, trigger or suppress apoptosis, or interfere with differentiation and developmental processes. An important tool to carry out such studies has been the GAL-UAS binary system for inducible gene expression [6]. The key feature of this system lies in a separable activator element mediated by the GAL4 gene whose expression is regulated by tissue-specific promoters and a $U A S$-target gene whose expression, in turn, is GAL4-dependent. Thus, expression can be regulated both temporally and spatially during development by crossing flies carrying a particular $U A S$-transgene to different $G A L 4$ lines.

In this paper, we have begun a large-scale functional analysis of human genes by assaying for heterologous gene activities in Drosophila. We have cloned and overexpressed 236 human genes in transgenic flies using the GAL4-UAS system [6, 7]. This represents so far only about $1 \%$ of the human genome. When the human genes were expressed individually by crossing to a panel of 8 different GAL4 drivers, 51 of the 236 transgenes were able to induce a reproducible phenotype. These results suggest the feasibility of a systematic and genome-wide screening of human gene functions by overexpression in Drosophila melanogaster.

\section{Results}

Constructing full-length human cDNAs for Drosophila germline transformation

To express the human genes in Drosophila, cDNAs were unidirectionally cloned into the P-element transformation vectors, $p U A S T$ [8] and $p U M A$. Both vectors include the mini-white ${ }^{+}$gene as a visible marker, two P-element terminal repeat sequences to mediate germline integration, and a UAS promoter for driving GAL4dependent gene expression of the cloned gene sequences. The restriction enzymes EcoRI and XhoI were used to linearize the $p U A S T$ vector for cloning. However, due to the lack of additional suitable cloning sites in this vector, the number of full-length cDNAs that we can clone into it is limited as both restriction enzymes used frequently cut in the human genome. To improve the efficiency of cloning full-length cDNAs, we designed the $p U M A$ vector based on the original $p U A S T$ plasmid. This new vector permits the ease of shuttling full-length inserts from the human cDNA library made in $p O T B 7$ by taking advantage of the restriction sites, PI-Sce I and I-Ceu I, for cloning. The strategy relies on the long recognition sites of these restriction enzymes, 39 bases for PI-Sce I and 26 bases for I-Ceu I, and the fact that these sites are absent in the human genome. The $p U M A$ vector significantly improves the cloning efficiency and retrieval of fulllength human cDNAs for making transgenic flies.

Once cloned into the appropriate vector, the P-element constructs are injected into $w^{1118}$ embryos to generate transgenic flies. The embryos were also co-injected with a helper plasmid ( $\Delta 2-3)$ which provides a source of transposase to allow integration into the genome. A germline integration event can be recovered by identifying adult flies in the F2 progeny that have red eyes as a result of carrying the mini-white ${ }^{+}$marker in the construct.

A total of more than 3500 random clones from the human cDNA library were picked and sequenced. The sequences were analyzed for homology and conserved domains by searching against the GenBank databases at the NCBI. We identified 236 clones with sequences indicative of potentially interesting functions, of which $26 \%$ were unknown and $74 \%$ were known genes. We generated a total of more than 1500 transgenic lines, with more than one line representing each human $\mathrm{cD}$ NAs. Additional information on these transgenic flies is presented in the Supplementary information, Table S1.

Overexpression of the human genes causes abnormal developmental phenotypes

The GAL4-UAS ectopic expression system has become a widely used and valuable tool for overexpression of transgenes in D. melanogaster. The GAL4 transcription factor can be expressed in different patterns, both temporally and spatially, by placing it under the control of various Drosophila promoters [8]. Once expressed, the GAL4 protein can then drive the expression of any transgene under a $U A S$ promoter. Furthermore, since GAL4 is a yeast transcription factor, which specifically recognizes only the $U A S$ sequence, it does not act on endogenous Drosophila promoters [8]. Among the large number of GAL4 drivers that are available on Flybase [9], we chose to use only those that are expressed in tissues where, if a phenotype is manifested, it can be easily observed and quickly scored under a dissecting microscope. This is an important consideration, especially for a screen adapted for high-throughput analysis. We selected a panel of eight different GAL4 drivers to cross to the collection of flies carrying the human transgenes (Table 1). The drivers have expression that occurs in patterns ranging from ubiquitous to being restricted to specific tissue, compartment, or cell type. The following are descriptions of the typical phenotypes, which are easily identifiable and scored, that we have observed using the various drivers affecting the development of the eye, the wing, the notum, and the scutella (bristles on the notum). 
Table 1 Phenotypes associated with GAL4-induced human gene expression

\begin{tabular}{llcl}
\hline GAL4 line & \multicolumn{1}{c}{ Expression pattern $^{1}$} & No. of genes & Main phenotype pattern \\
\hline ey-GAL4 & Eye-specific, dividing cells in the eye & 12 & Small eye \\
GMR-GAL4 & Eye-specific, differentiated cells in the eye & 28 & Rough eye \\
elav-GAL4 & Neuron specific & 14 & Extra or loss of bristles \\
en-GAL4 & Posterior part of imaginal discs & 14 & Lethal, loss of or extra vein \\
pnr-GAL4 & Mediodorsal parts of thoracic and abdominal segments & 29 & Cleft in the middle of notum \\
ptc-GAL4 & Dorsal mesothoracic disc & 18 & Extra or loss of bristle in scutellar \\
vg-GAL4 & Wing-specific & 12 & Nicked margin of wing \\
actin-GAL4 & Ubiquitous & 20 & Lethal \\
\hline
\end{tabular}

${ }^{1}$ See references $[8,13-16,22]$ for additional details.

Phenotypes associated with Drosophila eye development

The Drosophila eye is an indispensable organ for the viability of the fly. This makes it a particularly useful organ to observe for phenotypical consequences, whether caused by loss-of-function mutations or overexpression of a gene in the eye. Eye development proceeds in a stereotypical fashion where cells within the eye imaginal discs are specified, recruited, and differentiate in a sequential order leading to the highly precise structure of the adult eye (Figure 1A). Perturbations of signaling pathways that are involved in this process, such as those regulating cell proliferation, cell death, or differentiation, can lead to abnormal eye development. The GMR-GAL4 driver, for example, has expression mainly in the postmitotic cells that are undergoing differentiation in the eye imaginal disc [10]. Genes that are functional in this aspect of development will cause a rough eye phenotype when overexpressed with this driver as shown in Figure $1 \mathrm{~B}$.

In contrast, the ey-GAL4 driver (under the eyeless gene enhancer) has specific expression in dividing cells early in eye development [11]. Genes that can interfere with proliferative events at this stage generally will cause abnormal growth of the eye imaginal disc, leading to either a larger or a smaller adult eye. Some of the human transgenes under the eyeless expression pattern gave a small eye phenotype as shown in Figure 1G. Other transgenes can cause some eye tissues to undergo apoptosis (Figure 1C). Significantly, the Drosophila homologs of these same genes also cause a similar apoptotic phenotype when overexpressed likewise (Figure 1D). The results suggest that many human genes are likely to function in a physiologically relevant manner in flies to affect the same developmental process.

\section{Phenotypes associated with wing development}

The $v g$-GAL4 [12] is driven by the enhancer of the vestigial $(v g)$ gene, which has expression in the wing imaginal disc pouch, where the majority of growth occurs during wing development. Interference of one or more aspects of signaling required during this process can cause various abnormal wing development phenotypes [13]. For some transgenes, overexpression in a $v g$ pattern causes the loss or gain of wing venation (Figure 2B). Other transgenes appear to bring about the loss of tissues through apoptotic events leading to nicked or serrated margins in the adult wings (Figure 2C).

The en-GAL4 driver is specifically expressed in the posterior compartment of the wing disc tissue reflecting the expression pattern of the engrailed (en) gene [8, 14]. Phenotypes elicited by transgenes expressed in this way generally translate to defects in the posterior region of the adult wing. These also include changes in the size of the wing blade or the loss of bristles (Figure 4B).

The enhancer of the patched (ptc) gene utilized in $p t c$ GAL4 drives expression in a stripe of cells along the anterior/posterior $(\mathrm{A} / \mathrm{P})$ margin of the dorsal mesothoracic disc, which gives rise to the three adult structures: the notum, proximal wing, and the wing blade [15]. Some transgenes expressed in this A/P stripe give rise to phenotypes such as a complete or partial loss of bristles (Figure $2 \mathrm{E}$ and $2 \mathrm{~F}$ ). Similar phenotypes were also observed for some transgenes driven specifically in neurons using the elav-GAL4 driver, which has a pan-neural pattern of expression [8].

Phenotypes associated with the development of the notum and scutella

The driver pnr-GAL4 is expressed in a broad domain in the wing disc that contributes to the development of the presumptive notum. Forced expression of a number of transgenes in this manner results in adult flies with a cupped notum (Figure 2H) [16]. In some cases, bristle development on the notum is affected when particular transgenes are expressed using the elav-GAL4 driver. This phenotype is manifested as bristle clustering for a 

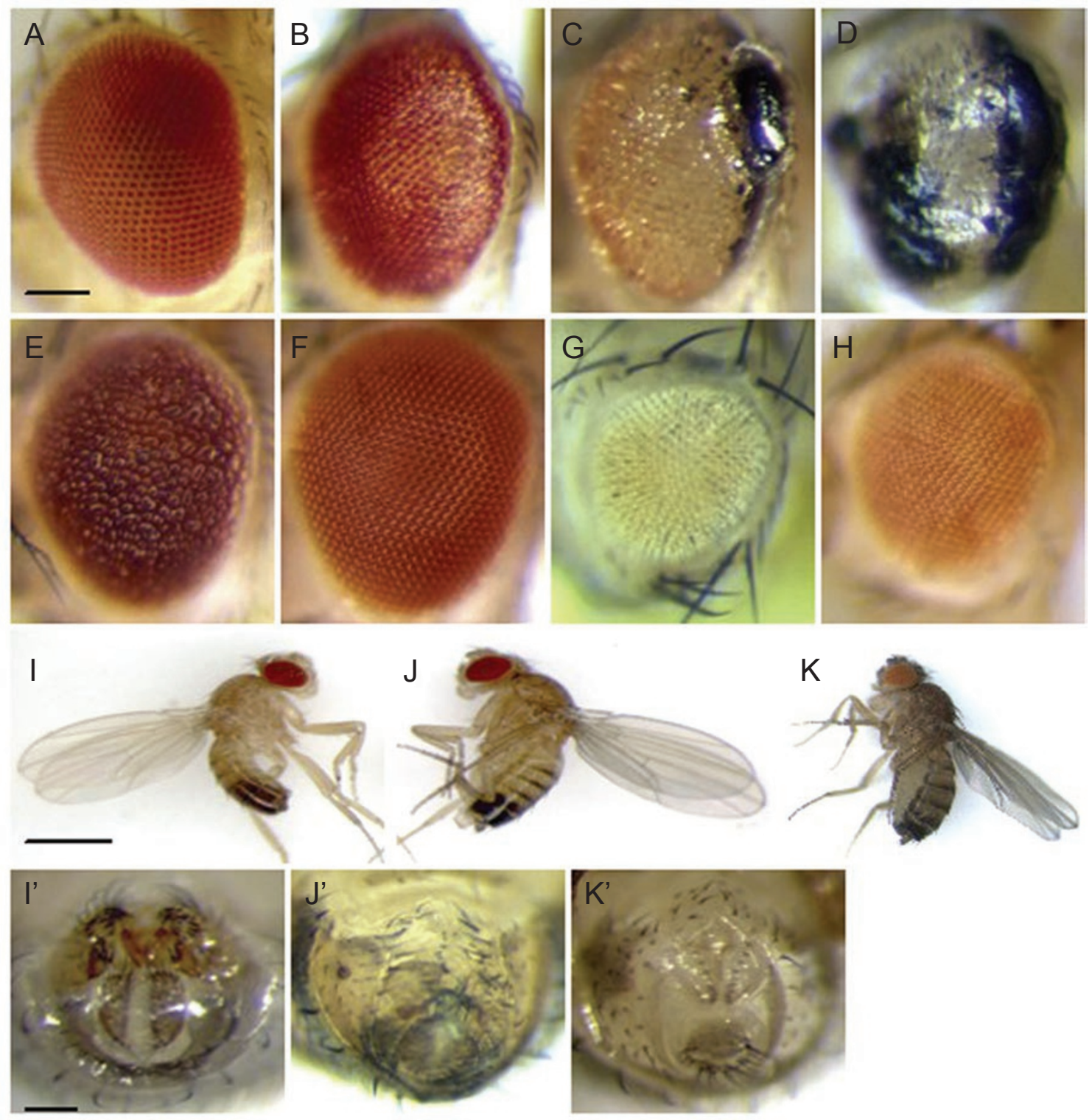

Figure 1 Mutant phenotypes induced by GAL4-dependent transgene expression. (A) Wild-type eye. (B) Rough eye phenotype induced in UAS-SFRS3; GMR-GAL4 flies. (C) Scars on the posterior part of eye of UAS-SFRS3; GMR-GAL4. (D) Scars on the posterior part of eye of UAS-dx16; GMR-GAL4. (E) p27-UAS; GMR-GAL4 (rough eye). (F) p27 ${ }^{\text {S10A }}$; GMR-GAL4 (rescued rough eye). (G) UAS-p27; ey-GAL4 (smaller eye). (H) p27 $7^{\text {S10A }}$; ey-GAL4 (partial rescue of small eye). (I) Wild-type male. (I') Normal male genitalia. (J) Male-like pigmentation in UAS-SFRS3: ptc-GAL4 flies. (J) Intersex genitalia. (K) Wild-type female. (K') Wild-type female genitalia. (A-H) Bar = $100 \mu \mathrm{m}$, (I-K) Bar = $1000 \mu \mathrm{m}$, (l'-K') Bar = $100 \mu \mathrm{m}$.

group of transgenes (Figure 2I), while others cause the loss of bristles (Figure 2J).

In summary, so far in our screen we have found 51 of the 236 human genes capable of eliciting a reproducible phenotype when overexpressed by all or a subset of the GAL4 drivers (Table 1). This suggests that these human genes are functional and can impinge on cellular and biochemical processes or events occurring in the heterologous context of fly development. Functional analyses of specific human transgenes are presented in the following sections.

The Ser10 phosphorylation site is required for p27Kip1 function in Drosophila

In mammals, p27(Kip1) is a key cell cycle regulator belonging to the Cip/Kip family of cyclin-dependent kinase inhibitor (CDKI), which also includes p21(Cip1) and p57(Kip2) [17-20]. It functions as a versatile CDKI 

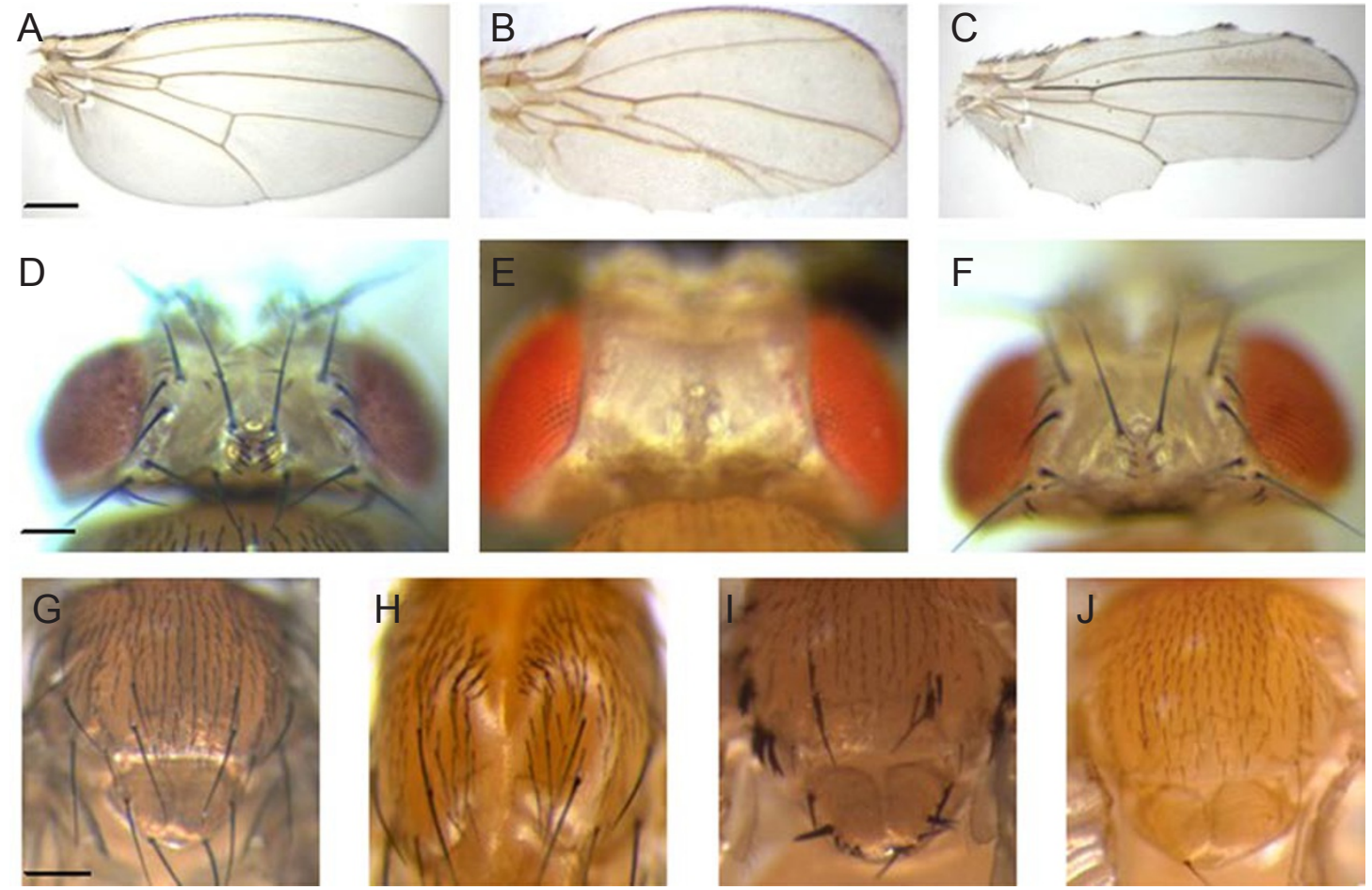

Figure 2 Phenotypes associated with overexpression in the wing, head, and notum. (A) Wild-type wing. (B) Lost vein and extended L5 in UAS-p27; ptc-GAL4 flies. (C) Nicked margins in UAS-SFRS3; vg-GAL4 flies. (D) Wild-type head. (E) Bristle loss in the head of UAS-SFRS3; elav-GAL4. (F) Lost post-ventral bristles in MORC 1A11; ptc-GAL4 flies. (G) Wild-type notum and scutella. (H) Cupped notum phenotype. (I) Extra bristles in the scutella of MORC 1M19; elav-GAL4 flies. (J) Lost bristle in the notum of MORC 1W04; elav-GAL4 flies. (A-C) Bar $=250 \mu \mathrm{m},(\mathrm{D}-\mathrm{F})$ Bar $=100 \mu \mathrm{m},(\mathrm{G}-\mathrm{J})$ Bar $=200 \mu \mathrm{m}$.

to block the G1/S transition and to negatively regulate cell cycle progression by inhibiting CDK activities [21]. Since the loss of function results in unchecked cell proliferation, $p 27$ has been regarded as a tumor suppressor gene $[22,23]$. This is consistent with the finding that reduction of its function or level is highly correlated with tumor prognosis and development. Thus, $p 27$ has become an important focal point in cancer research, and a strategic target for diagnosis and treatment.

However, in view of the traditional definition of a tumor suppressor, gene mutation or silencing at the p27 locus is rarely found. Evidence points to posttranscriptional regulation as the major mechanism by which p27 activity is modulated in the context of tumor development. Several means of regulation have been observed involving translational control, phosphorylation, proteolytic mechanisms, and subcellular transport [24]. Recently, the discovery of several new domains and phosphorylation sites in p27 has shed new light on its function. Although there are three phosphorylation sites (S10, T157, and T187), there has been much interest in the phosphorylation of S10, which affects the stability and nuclear export of p27 [25]. It has been suggested that
S10 phosphorylation plays a key role in regulating the tumor suppressor function of $\mathrm{p} 27$.

We wish to address the functional significance of Ser10 in our Drosophila transgenic model. Initially, to test whether the human $p 27$ gene is functional in the fly, we obtained five independent transgenic lines for overexpressing the gene. Using various GAL4 drivers, we showed that $p 27$ is functional in a variety of developmental contexts. For example, overexpression in the eye by $G M R-G A L 4$ or ey-GAL4 induces either a rough or a small eye phenotype, respectively (Figure $1 \mathrm{E}$ and $1 \mathrm{G})$. Constitutive expression of $p 27$ using the actinGAL4 driver, however, results in lethality. To determine whether the S10 phosphorylation site has any functional significance, we mutated the Ser to Ala to make the $p 27^{S 10 A}$ transgene. In contrast to wild-type $p 27$, expression of the $p 27^{S 10 A}$ transgene driven by GMR-GAL4 or ey-GAL4 induces a barely perceptible phenotype that is hard to distinguish from the wild-type eye (Figure 1E$1 \mathrm{H})$. The results suggest that S10 phosphorylation contributes significantly to the function of $p 27$, even in this heterologous context. 
The SFRS3 splicing factor affects sexual differentiation of Drosophila genitalia

Approximately $40-60 \%$ of the genes in humans encode alternatively spliced transcripts. The evolution of alternative splicing mechanisms allows for greater functional diversity and regulation [26]. The splicing reaction is carried out by the spliceosome, which is a large complex made of small nuclear ribonucleoprotein particles (snRNPs) as well as non-snRNP proteins that assemble at specific sites on the pre-mRNA. Splice site selection is achieved with additional factors that are recruited to the spliceosome. Among these are members of a highly conserved family of proteins, the SR (Ser/Argrich) proteins. They have been shown to play important roles in both the general and tissue-specific regulation of alternative splicing of pre-mRNAs and their maturation. In Drosophila, several SR proteins have been wellcharacterized. In particular, the TRA, TRA2, and RBP1 proteins form complexes with the spliceosome which bias female-specific splicing of the doublesex $(d s x)$ premRNA for sex determination during development.

Among the human transgenes used in our screen, we have identified a gene called SFRS3 (ㅁplicing Factor $\underline{\text { Ar- }}$ ginine/Serine-rich 3), which also belongs to the SR protein family. We tested the functional consequences of its expression in the fly. Interestingly, the ptc-GAL4 driver induces a phenotype displaying defects in sexual differentiation of the genitalia where characteristics of both sexes were observed (Figure 1J). For example, female flies expressing SFRS3 show the appearance of malelike clasper bristles together with reduced number of vaginal bristles, which normally are present in two rows flanking the female genitalia. Additional defects include abnormal development of the anus (Figure $1 \mathrm{~J}^{\prime}$ ). In a weakly expressing transgenic line, the elav-GAL4 driver induces a male-like pigmentation pattern in the posterior abdominal segment of a female, which normally lacks such pigmentation (Figure $1 \mathrm{~K}$ and $1 \mathrm{~K}^{\prime}$ ). This female is also sterile.

We cloned and made an overexpression construct for $d x 16$, the Drosophila homolog of SFRS3 [27]. This has enabled us to show that $d x 16$ and SFRS3 are likely to have similar functions during development. For example, both transgenes gave a similar eye phenotype when they were overexpressed using the GMR-GAL4 driver (Figure $1 \mathrm{C}$ and 1D).

The human ribosomal protein L8 affects cell size regulation in Drosophila

Ribosome biogenesis and protein biosynthetic pathways play critical roles in the development and growth of organisms. Generally, an increase in ribosome biogenesis often stems as a consequence of an increase in cellular proliferation during tissue and organ growth. In contrast, abrogating or reducing the efficiency of protein synthesis often leads to reduced growth rates and survival. In Drosophila, a class of dominant mutations called Minute display phenotypes that, indeed, reflect a reduction in growth rate at both cellular and organismal levels. The mutations are in genes encoding ribosomal proteins (RPs).

One of the human transgenes in our screen encodes the RP RPL8. When the transgene is expressed with GMR-GAL4, the adult flies have eyes that are smaller and appear flattened (Figure 3B). The apparent defect is not due to a reduction in the number of ommatidia, but rather due to a reduction in their size $(\sim 26 \%$ decrease in size, $n=10$, Figure $3 \mathrm{~B}^{\prime}$ ). Furthermore, we eliminated the possibility that the phenotype could be due to a problem in differentiation by staining the larval eye discs with anti-Elav, a neuronal-specific antibody [28, 29]. Consistent with the adult eye phenotype, the photoreceptor clusters are also smaller than those of control (boxed regions in Figure $3 \mathrm{G}^{\prime}$ and $3 \mathrm{H}^{\prime}$ ), but they undergo normal neuronal differentiation in the posterior of the eye discs (Figure $3 \mathrm{G}, 3 \mathrm{G}^{\prime}, 3 \mathrm{H}$, and $\left.3 \mathrm{H}^{\prime}\right)$. Thus, the induced small eye phenotype is entirely due to a reduction in cell size during eye development.

We were entirely surprised by this observation since, if RPL8 is functionally conserved, we would have expected an opposite phenotype, i.e. an increase in cell size correlating with greater efficiency of protein synthesis when human RPL8 is overexpressed. We sequenced the $R P L 8$ transgene construct and found a frame shift mutation at residue G710, which leads to a truncation of the 257 amino acid full-length protein at Glycine 236 and a fusion of an additional 52 amino acid residues to the C-terminus (Supplementary information, Figure S1). Many truncated proteins often exhibit dominant negative effects by interfering with the normal function of the endogenous wild-type protein or protein complexes. By definition, a dominant negative mutant can be suppressed by increasing the level of wild-type function. To test this hypothesis, we generated a transgene to co-express the wild-type Drosophila RPL8 together with the truncated human $R P L 8$. Indeed, the small eye phenotype caused by the truncated human $R P L 8$ is completely rescued with Drosophila RPL8 (Figure 3D and 3D'). The overexpression of Drosophila RPL8 alone, however, has no effect on the ommatidia size (Figure $3 \mathrm{C}$ and $3 \mathrm{C}^{\prime}$ ). Thus, the truncated human RPL8 gene behaves genetically as a dominant negative allele, which will be denoted here as human $R P L 8^{D N}$.

The Drosophila RPL8 is a single copy gene and appears to be the only ortholog of the human RPL8. Both 
genes are highly conserved with an overall $79 \%$ amino acids identity and $87 \%$ amino acids similarity (Supplementary information, Figure S1). Drosophila RPL8 is ubiquitously expressed during all stages of Drosophila development, which possibly suggests a general role in protein synthesis [30]. In order to further define the function of Drosophila RPL8, we designed an RNA interference construct (Drosophila RPL $8^{R N A i}$ ) to downregulate the endogenous Drosophila RPL8 gene expression. When expressed using GMR-GAL4, the Drosophila RPL $8^{R N A i}$ transgene causes the same cell size reduction phenotype

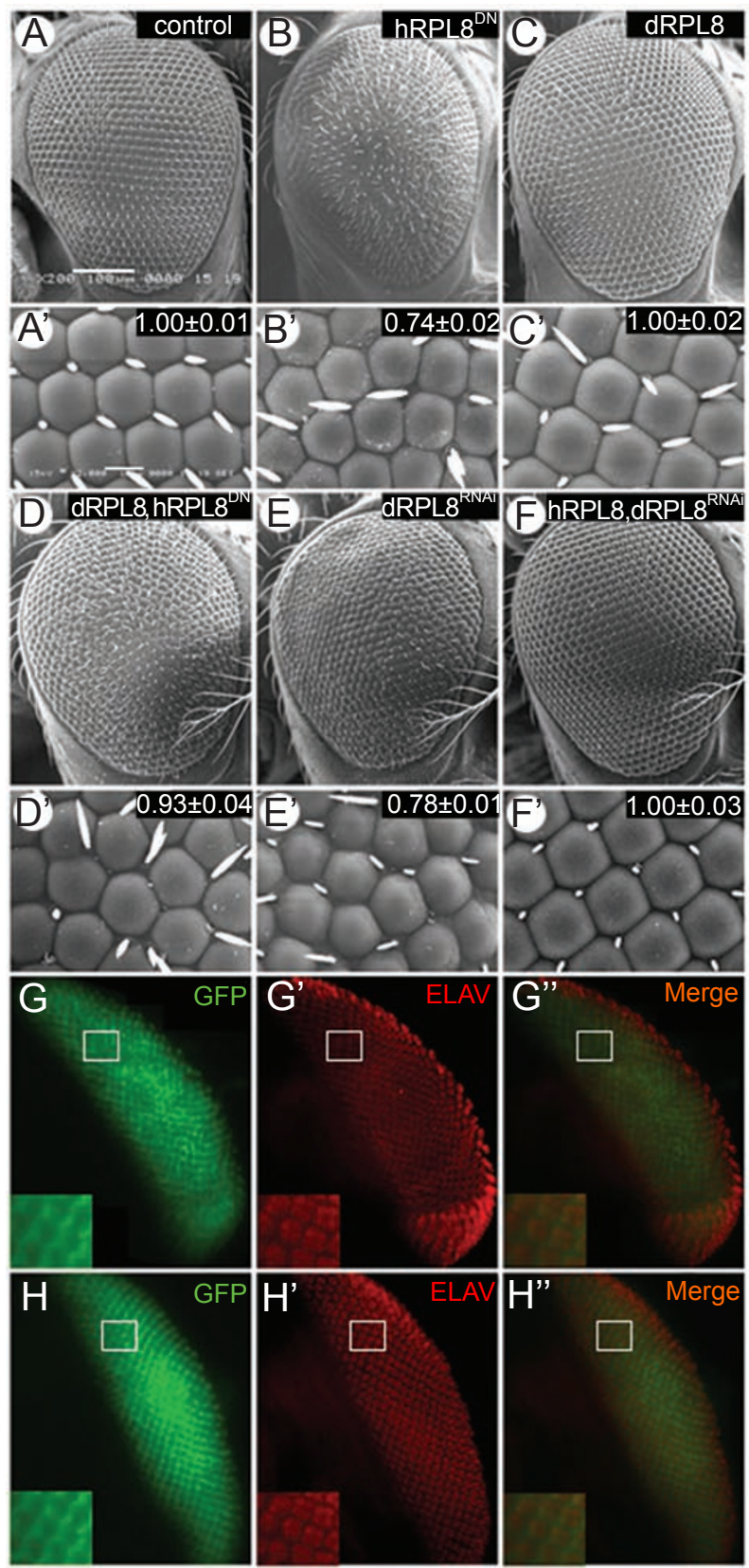

as observed with human $R P L 8^{D N}$ (Figure 3E and 3E'). We co-expressed the wild-type Drosophila RPL8 with Drosophila $R P L 8^{R N A i}$ to test whether the small eye phenotype can be rescued. Indeed, the phenotype is completely reverted to wild type (data not shown). The result suggests that the effect of the RNAi downregulation is specific to Drosophila RPL8. Furthermore, we also co-expressed the wild-type human RPL8 together with Drosophila $R P L 8^{R N A i}$ and found the RNAi phenotype could be completely rescued (Figure $3 \mathrm{~F}$ and $3 \mathrm{~F}^{\prime}$ ). The replacement of the human RPL8 gene for the loss of Drosophila RPL8, thus, strongly supports the functional conservation of the RPL8 genes and their role in regulating cell size in Drosophila and humans.

Drosophila RPL8 regulates cell size in multiple tissues during development

Since Drosophila RPL8 is ubiquitously expressed, we wish to determine whether it also functions similarly in regulating cell size in other tissues. However, the Drosophila $R P L 8^{R N A i}$ construct causes embryonic lethality when driven by different $G A L-4$ drivers other than the $G M R-G A L 4$, presumably due to leaky expression. To overcome this problem, we tried the dominant negative human $R P L 8^{D N}$ construct instead, which did not result in lethality. We induced the expression of human $R P L 8^{D N}$ using the en-GAL4 driver, which limits expression to the posterior compartment of the wing disc. The phenotype was analyzed by counting cell numbers and measuring areas in the posterior and anterior compartments of the adult wing, and the results compared with those obtained

Figure 3 RPL8 regulates ommatidia size but not cell differentiation and pattern formation. Scanning electron micrograph of an adult Drosophila eye and close-up of the ommatidia (A and A', respectively). The inset in ( $\mathbf{A}^{\prime}$ ) indicates the value for the cell size of an ommatidium normalized against wild type. Adult eyes of flies expressing various human and Drosophila RPL8 constructs as noted in the insets (B-F). (B'-F') Corresponding closeups of ommatidia from flies in (B-F). Insets indicate normalized differences in cell size. Genotypes are as follows: GMR-GAL4/+ (A, A); GMR-GAL4/UAS-humanRPL8 ${ }^{D N}$ (B, B ); GMR-GAL4/ UAS-DrosophilaRPL8 (C, C); GMR-GAL4, UAS-humanRPL8 ${ }^{D N}$, UAS-DrosophilaRPL8 (D, D ); GMR-GAL4/UAS-DrosophilaR$P L 8^{R N A i}$ (E, E); GMR-GAL4, UAS-DrosophilaRPL8 $8^{R N A i} / U A S$ humanRPL8 (F, F ). GMR-GAL4 directed expression of GFP in wild-type third instar eye discs (G), Elav staining of the same disc (G), and a merged image (G'), demonstrating the overlap between the GMR expression pattern and the region of neuronal cell differentiation. Overexpression of mutant human RPL8 in the posterior region of third instar larvae eye discs has no effect on these staining patterns ( $\left.\mathbf{H}^{\prime}, \mathbf{H}^{\prime \prime}\right)$. Genotypes are: $G M R$ GAL4/+; UAS-GFP/+ (G-G"); GMR-GAL4/UAS-humanRPL $8^{D N}$; UAS-GFP/+ (H-H"). (A-F) Bar $=100 \mu \mathrm{m}\left(\mathbf{A}^{\prime}-\mathbf{F}^{\prime}\right)$. Bar $=10 \mu \mathrm{m}$. 

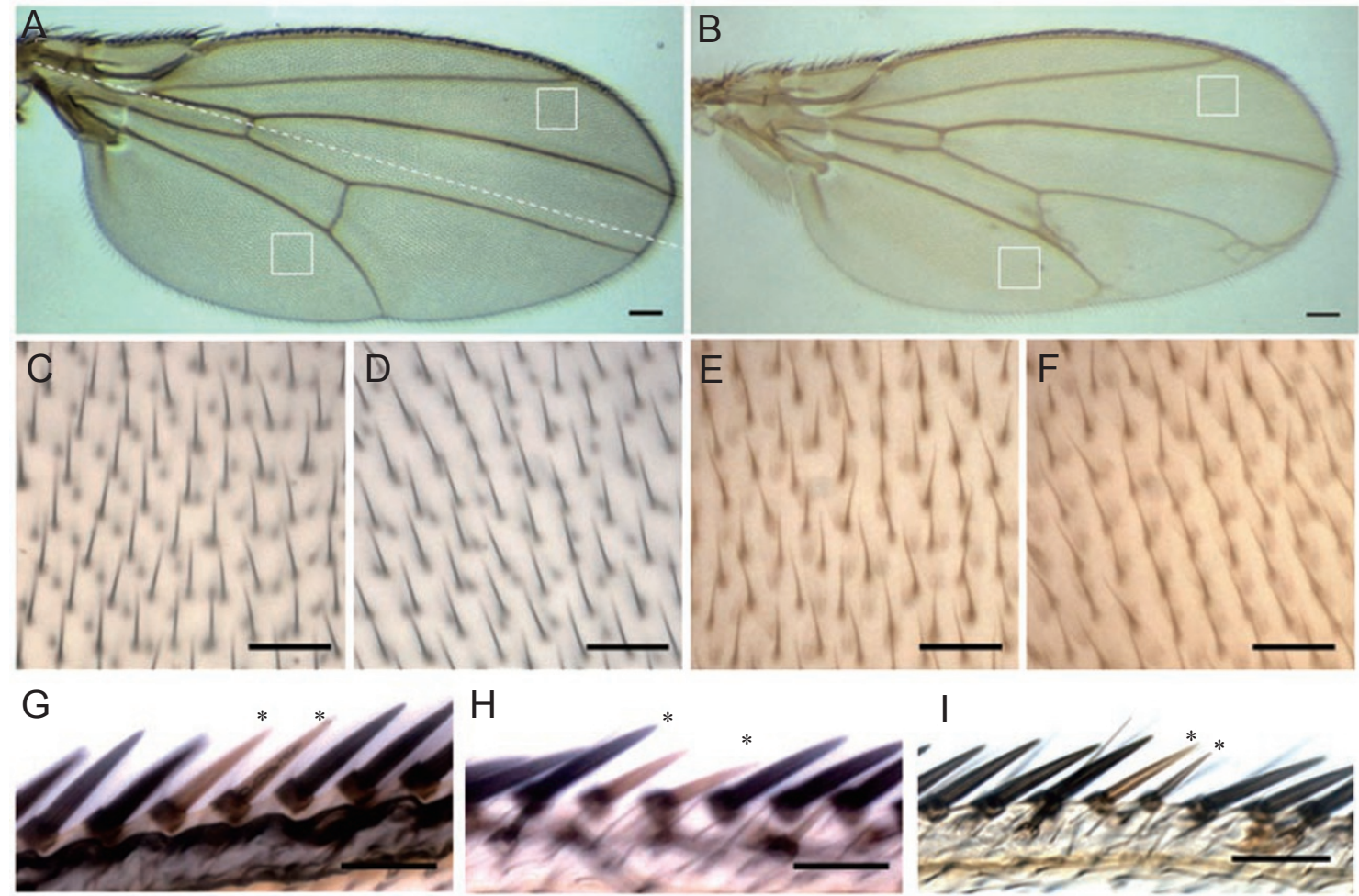

Figure 4 Overexpression of mutant humanRPL8 decreases the cell size in the adult fly wing. Light micrograph of adult female wings of wild-type $w^{1118}$ flies $(\mathbf{A})$ or en-GAL4/UAS-humanRPL8 $8^{D N}(\mathbf{B})$. The dotted line in $(\mathbf{A})$ denotes the anterior/posterior wing margin. (C, D) Enlarged views of the boxed regions in (A) showing anterior (top half) and posterior (bottom half) areas containing wild-type tissues. (E, F) Corresponding close-ups for mutant wing in (B). (G) Wing margin of wild-type FLPout clones. Asterisks mark bristles corresponding to cells with activated GAL4 expression, seen as loss of the yellow pigment $(y-)$. $(\mathbf{H}, \mathrm{I})$ Flip-out clones of cells overexpressing humanRPL8 $8^{D N}\left(y\right.$, hsFLP; Actin>y+>GAL4/UAS-humanRPL $\left.8^{D N}\right)$ or DrosophilaRPL $8^{R N A i}$ ( $y w$ hsFLP; Actin>y+>GAL4/UAS-DrosophilaRPL $8^{\text {RNAi }}$ ), respectively. Bar $=50 \mu \mathrm{m}$.

for wild-type wings (Supplementary information, Table $\mathrm{S} 2)$. The overexpression of human $R P L 8^{D N}$, indeed, not only caused a reduction in cell size but also reduced cell number and consequently the overall wing size (Supplementary information, Table S2). These changes were limited to the expression domain of en-GAL4 (Figure $4 \mathrm{~A}-4 \mathrm{~F})$.

We also performed clonal analysis in order to clearly compare the differences in cell size between neighboring mutant and wild-type cells. The "FLIP-out" technique was used to generate marked overexpression clones for either human $R P L 8^{D N}$ or Drosophila $R P L 8^{R N A i}$ in the anterior margin of the wing [22]. Clones that overexpress the transgene would appear yellow since they lack expression of the yellow ${ }^{+}$marker. We compare neighboring mutant and wild-type wing margin bristles by looking at their shafts, which has been used as an indication of cell size of the underlying cells responsible for their formation [31]. We found that wing margin bristle cells expressing either human $R P L 8^{D N}$ or Drosophila $R P L 8^{R N A i}$ have a $20 \%$ reduction in size (Figure $4 \mathrm{G}-4 \mathrm{I}$ ). The result is consistent with previous observations in the eye, and indicates that Drosophila RPL8 has a role in the regulation of cell size in multiple issues throughout development.

Drosophila RPL8 functions downstream of the insulin signaling pathway

The insulin signaling pathway has been demonstrated to regulate many aspects of biological processes including cell proliferation, cellular growth, and survival. This pathway is conserved in Caenorhabditis elegans, Drosophila, and mammals [32]. Interestingly, mutations in the Drosophila S6 Kinase $(S 6 K)$, which functions as a positive regulator downstream of the pathway, cause phenotypes similar to those of human $R P L 8^{D N}$ and Drosophila $R P L 8^{R N A i}$ [33]. The similarity in cell growth defects suggests the involvement of a common pathway affecting ribosome biosynthesis and protein synthesis, perhaps through aberrant insulin signaling. Therefore, 

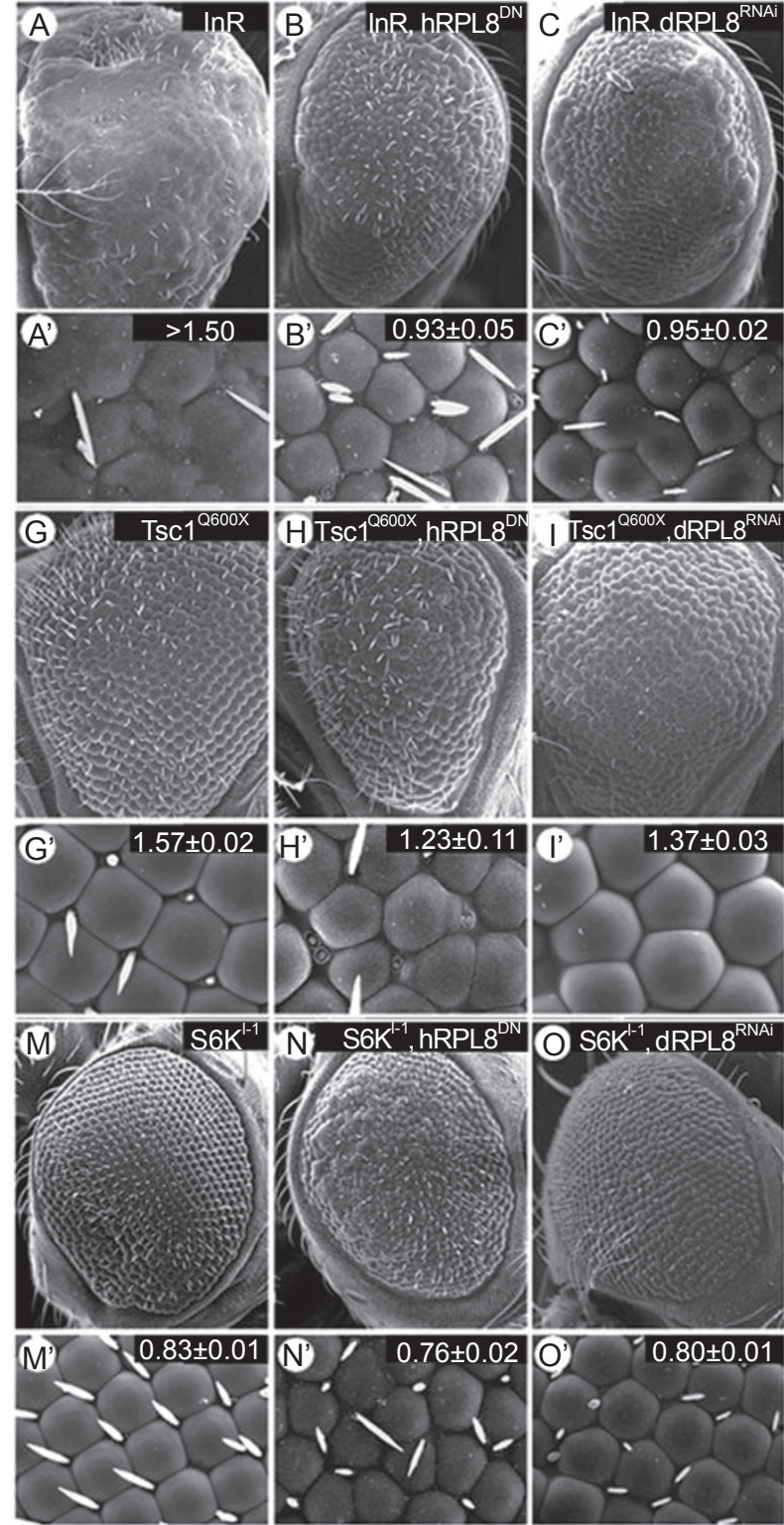
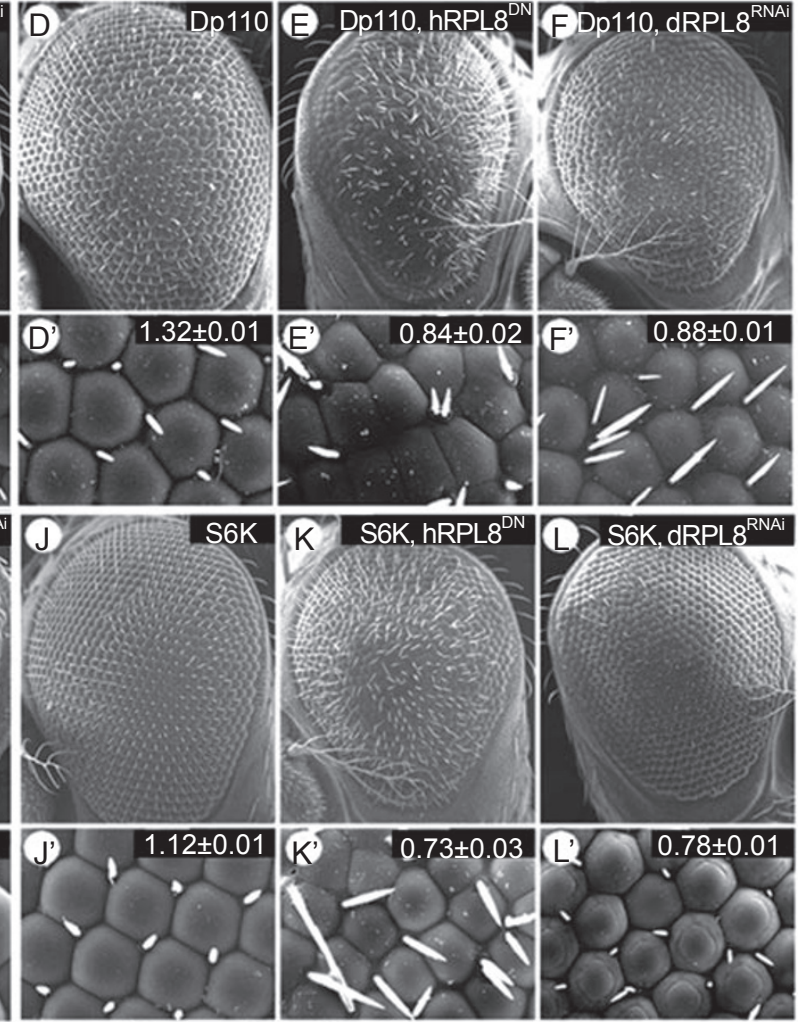

Figure 5 Genetic analysis of RPL8 function in the insulin signaling pathway. SEMs of female adult eyes and enlarged areas showing ommatidia of flies with the following genotypes: GMR-GAL4, UAS-InR/CyO (A, A); GMR-GAL4, UAS-InR/UAS$\operatorname{humanRPL8}^{D N}$ (B, B); GMR-GAL4, UAS-InR/UAS-DrosophilaRPL8 ${ }^{R N A i}$ (C, C); GMR-GAL4/UAS-Dp110 (D, D); GMR-GAL4, UAS-humanRPL8 $8^{D N} / U A S-D p 110$ (E, E); GMR-GAL4, UAS-DrosophilaRPL8 ${ }^{R N A i} / U A S-D p 110$ (F, F ); y w eyFLP/y w; CyO/+; FRT 82B GMR-hid CL3R/FRT 82B TsC1 ${ }^{\text {Q600X }}$ (G, G); y w eyFLP/y w; GMR-GAL4, UAS-humanRPL8 $8^{D N} /+; F R T$ 82B GMR-hid CL3R/FRT 82B Tsc1 ${ }^{\text {Q600X }}(\mathbf{H}, \mathbf{H})$; y w eyFLP/y w; GMR-GAL4, UAS-DrosophilaRPL8 ${ }^{R N A i} /+;$ FRT 82B GMR-hid CL3R/FRT 82B Tsc1 ${ }^{\text {Q600X }}$ (I, I); UAS-DrosophilaS6K/CyO;GMR-GAL4/+ (J, J); UAS-DrosophilaS6K/UAS-humanRPL8 ${ }^{D N}$; GMR-GAL4/+ (K, K); UAS-DrosophilaS6K UAS-DrosophilaRPL8 ${ }^{R N A i}$; GMR-GAL4/+ (L, L'); y w eyFLP/y w; CyO/+; GMR-hid CL3L FRT 80B/ DrosophilaS6K $K^{l-1}$ FRT $80 B$ (M, M); y w eyFLP/y w; GMR-GAL4, UAS-humanRPL8 $8^{\text {NN }}$; GMR-hid CL3L FRT 80B/DrosophilaS6K $K^{1-1} F R T 80 B$ (N, N); y w eyFLP/y w; GMR-GAL4, UAS-DrosophilaRPL8 $8^{\text {RAAi } /+; ~ G M R-h i d ~ C L 3 L ~ F R T ~ 80 B / D r o s o p h i l a S 6 K ~}$ I $^{1-1}$ FRT $80 B(\mathbf{0}, \mathbf{0})$.

we performed genetic interaction experiments to test whether Drosophila RPL8 might be a target in the insulin signaling pathway.

Increasing activity of the insulin signaling pathway affects cellular growth, which leads to an increase in cell size. For example, GMR-Gal4-induced expression of the Drosophila Insulin Receptor (InR) or Dp110, the Drosophila homolog of phosphoinositide 3-kinase (PI3K), 
leads to an increase in ommatidia size (Figure 5A and $5 \mathrm{D}$, respectively). This phenotype can be suppressed by the co-expression of either Drosophila $R P L 8^{R N A i}$ or hu-

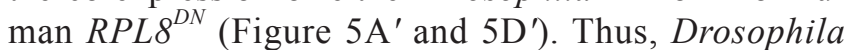
$R P L 8$ appears to act downstream of Drosophila InR and $D p 110$, since their activities are dependent on the function of Drosophila RPL8.

Removing $T s c 1$ function, which is a negative regulator of the insulin signaling pathway, also causes an increase in ommatidia size (Figure 5G and 5G') [29]. Since Tsc1 mutations are lethal, we generated $T s c 1$ mutant mosaic eyes through the induction of somatic clones. In addition, we utilized the EGUF/hid cell ablation technique to remove all wild-type cells in the mosaic such that the entire eye is mutant for $T s c 1$. Consequently, this leads to an increase in size of both the eye and head capsule [34]. The phenotype can be suppressed by the co-expression of either Drosophila RPL ${ }^{R N A i}$ or human $R P L 8^{D N}$ (Figure $5 \mathrm{H}, 5 \mathrm{H}^{\prime}, 5 \mathrm{I}$, and 5I'), suggesting that Drosophila RPL8 also functions downstream of $T s c 1$.

Drosophila $S 6 K$ is the most downstream component of the insulin signaling pathway. Its overexpression by GMR-GAL4 results in eyes which are slightly bigger than wild type (Figure $5 \mathrm{~J}$ and $5 \mathrm{~J}^{\prime}$ ). The phenotype can be suppressed by the co-expression of Drosophila RPL $8^{R N A i}$ or human $R P L 8^{D N}$ (Figure $5 \mathrm{~K}, 5 \mathrm{~K}^{\prime}, 5 \mathrm{~L}$, and $5 \mathrm{~L}^{\prime}$ ), which suggests that Drosophila RPL8 is downstream of Drosophila $S 6 K$. This is an intriguing finding since the $\mathrm{S} 6 \mathrm{RP}$ is the only known target of the S6 kinase. To further con- firm the genetic interaction between Drosophila $S 6 K$ and Drosophila RPL8, we expressed Drosophila $R P L 8^{R N A i}$ or human $R P L 8^{D N}$ in EGUF/hid-induced mosaic clones that are mutant for Drosophila S6K. Mosaic eyes consisting primarily of Drosophila $S 6 K$ mutant ommatidia were slightly smaller than wild type due to a decrease in ommatidia size (Figure $5 \mathrm{M}$ and $5 \mathrm{M}^{\prime}$ ). However, flies with reduced activity for both Drosophila $S 6 K$ and Drosophila $R P L 8$ had an even smaller eye (Figure $5 \mathrm{~N}, 5 \mathrm{~N}^{\prime}, 5 \mathrm{O}$, and $5 \mathrm{O}^{\prime}$ ). Interestingly, this double mutant phenotype is comparable to those of flies in which only Drosophila RPL8 activity is reduced. Together these results support the hypothesis that Drosophila RPL8 acts downstream of the insulin signaling pathway and might have additional functions affecting translational efficiency and output, which are required for cellular growth.

\section{Drosophila RPL8 genetically interacts with Drosophila Myc in growth regulation}

In addition to the insulin signaling pathway, signaling that impinges on Drosophila Myc activity also has a role in cell growth regulation [35]. GMR-GAL4-induced expression of Drosophila Myc causes an enlarged eye phenotype (Figure 6B and 6B'). This phenotype can be partially suppressed with a reduction in Drosophila $R P L 8$ activity (Figure 6C, 6C', 6D, and 6D'). This finding indicates that Drosophila RPL8 may also function downstream of or parallel to $m y c$ in the regulation of cell growth.
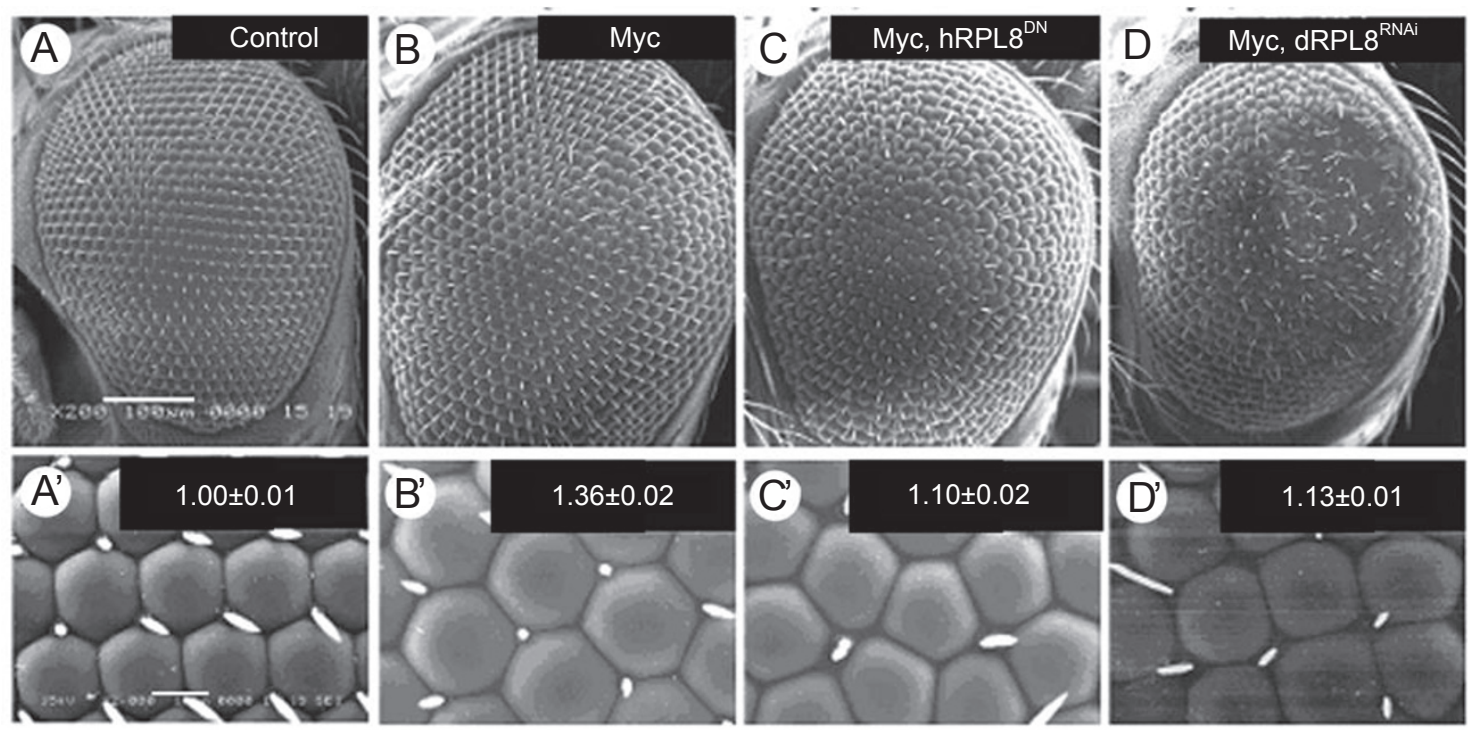

Figure 6 Loss of RPL8 function negatively affects the activation of Drosophila Myc pathway. SEMs of female adult eyes and enlarged areas showing ommatidia of flies with the following genotypes: GMR-GAL4/CyO (A, A); GMR-GAL4, UAS-myc/ CyO (B, B ); GMR-GAL4, UAS-myc/ UAS-humanRPL8 ${ }^{D N}$ (C, C'); GMR-GAL4, UAS-myc/ UAS-DrosophilaRPL8 ${ }^{R N A i}$. (A-D) Bar $=100 \mu \mathrm{m},\left(\mathrm{A}^{\prime}-\mathrm{D}^{\prime}\right)$ Bar $=10 \mu \mathrm{m}$. 


\section{Discussion}

Even though there is a large order of complexity between human and flies, the underlying cellular and biochemical processes are highly conserved. Indeed, components of major signaling pathways were first identified in D. melanogaster and C. elegans. Genetic studies in these model organisms, which would not have been as feasible in mammals and humans, have provided important mechanistic and functional insights into how these pathways regulate various cellular and developmental processes. Among the arsenal of genetic tools available in the fly is the ability to overexpress heterologous genes in vivo. These studies are particularly useful for indentifying potential human gene functions since they provide functional clues from abnormal phenotypes induced by overexpression of a particular gene. The phenotypes serve as indications that the heterologous genes are capable of perturbing the function of one or more conserved signaling pathways in flies. Together with what can be learned from the Drosophila homologs, such observations enable further investigation into the role and function of the human gene counterparts.

The yeast GAL4 transcriptional activator has been widely used in many heterologous systems to drive target gene expression. By using various promoters that are developmentally regulated, constitutive or conditional, GAL4 expression can be controlled temporally and spatially in various tissues and at various stages of development. Placing target genes downstream of the UAS promoter, which contains GAL4-binding sites, allows for GAL4-dependent gene expression. The utility of the $G A L 4 / U A S$ binary system can also be extended to express genes that might be lethal to the organism, since the target gene would be silent in the absence of GAL4. The flexibility of using different GAL4-expressing lines enables the lethal gene product to be expressed briefly or at a specific developmental stage when the biological process of interest is being studied. Among its other uses, this system is an especially valuable tool for studying target genes that are required in multiple developmental processes or act at several stages in development since their roles can be dissected by restricting GAL4-dependent expression to specific cells, tissues, or particular stage of development.

In this study, a panel of eight GAL4 drivers were used for inducing target gene expression in the eye, nervous system, various imaginal disc compartments, and tissue domains, as well as ubiquitously (actin-GAL4). The choice of these drivers is based on the fact that expression of exogenous genes in these patterns generally induces phenotypes that are easily identifiable and quickly scorable under a dissecting microscope. This is a key factor in a genome-wide screen where many transgenes need to be tested. We have observed that the GMR-GAL4 and $p n r-G A L 4$ drivers tend to induce a much stronger expression than the other GAL4 lines. Consequently, tissues expressing a transgene under GMR-GAL4 and pnr-GAL4 are more sensitive to phenotypic induction. The $v g-G A L 4$ and en-GAL4 are the weakest of the eight GAL4 drivers and, therefore, tend to provide fewer positives in our screen (Supplementary information, Table S1). We have produced and used multiple transgenic lines for each transgene in our analysis. Nevertheless, we found that two independent transgenic lines for each transgene are sufficient for the initial screen.

After the primary screen, human candidate transgenes with interesting phenotypes are chosen for further analysis. By using additional GAL4 drivers one can dissect the cellular and developmental basis of an overexpression phenotype, for example, by examining whether cell proliferation, cell growth, differentiation, or apoptosis are affected. Furthermore, one powerful application of an overexpression phenotype is that one can assay for dominant modifiers by crossing to known mutations in signaling pathways. Such an approach facilitates the identification of the potential role of a particular human gene in different biological processes and developmental pathways.

In our study, we have randomly selected 236 human genes to construct transgenic lines in the fly. Transformation and overexpression are mediated by the P-elementbased vectors, $p U A S T$ and $p U M A$; the latter was designed for efficient cloning of full-length human cDNA sequences. When driven by different GAL4 lines, 51 out of 236 human genes showed detectable overexpression phenotypes. The success of this approach provides the feasibility of adapting the GAL4/UAS binary system to a large-scale screen for uncovering human gene functions.

Previous studies have shown that $2-7 \%$ of the Drosophila genes can be induced to cause developmental phenotypes when they are ectopically expressed in a given spatial or temporal pattern [12, 35-38]. Considering that $61 \%$ of human genes are conserved in the fly [3], we had estimated that $5 \%$ of the human genes can be expected to show some phenotypical consequences when overexpressed in Drosophila. Surprisingly, as our results have shown, approximately $21 \%$ of the human genes that we have tested show detectable phenotypes. This higher than expected result may be explained by the fact that, rather than using a single driver, we had used a panel of different GAL4 lines to induce gene expression, each of which varies in transcriptional efficacy. Also, multiple transgenes for each cDNA clone is also an important 
consideration, since their level of expression depends, in many cases, on the position of their insertion in the fly genome. Alternatively, the higher number of inducible phenotype may reflect the biological differences between humans and Drosophila. It remains to be determined whether all or some of the phenotypes are due to physiologically relevant biochemical functions or that some of the human gene products can have neomorphic activities in Drosophila tissues.

An interesting case is the phenotypic analysis of the human RPL8 transgene. Given its role as a RP, we had expected the overexpression of human $R P L 8$ to have a positive influence on growth rather than the opposite effect of causing reduced cell size. Sequencing the original transgene construct revealed that we had actually expressed a truncated form of the protein instead, which functions as a dominant negative mutant as revealed by further genetic analysis. Nevertheless, the human RPL8 overexpression phenotype still provided insight into its in vivo function in the control of cell size and, as a result, organ size.

We extended the phenotypic analysis to the Drosophila RPL8 homolog using an RNAi construct to downregulate its function. Growth defects were seen in both the eye and the wing, suggesting that Drosophila RPL 8 may be a general regulator of cell size in multiple tissues. The lethality caused by the ubiquitous expression of Drosophila $R P L 8^{R N A i}$, and apoptosis when expressed in the eye, strongly suggests that Drosophila RPL 8 is likely to be an essential gene. Interestingly, the cell death phenotype, but not the defect in cell size, can be rescued by the co-expression of the $\mathrm{p} 35$ cell death inhibitor. The cell death aspect of the phenotype may be a consequence of lowered protein synthesis, which, in turn, might result in diminished levels of negative regulators of apoptosis. In contrast to the loss-of-function phenotypes, overexpression of wild-type Drosophila RPL8 (Figure 3C and 3C') or human $R P L 8$ had no effect on ommatidia size. Since ribosomal complexes consist of stoichiometric number of RP s and other translation factors, it is likely that an increase in any single component might not affect complex formation and function. On the other hand, mutations in RP s, such as those encoded by the Minute genes, show dosage-dependent growth phenotypes. A reduction in gene dosage by half leads to the classic small fly phenotype. These genes, thus, show haploinsufficiency and, therefore, are manifested as dominant mutations.

The molecular mechanism of organ size control is not fully known. Studies in Drosophila indicate that the relationship between cell size and organ size is complex. If one considers the total mass of an organ to reflect its size, then, it becomes clear that any changes to cell size would affect organ size. Indeed, this is true in some instances; however, there are cases where changes in cell size do not affect organ size $[39,40]$. The result we obtained with overexpressing human $R P L 8^{D N}$ in the eye suggests that organ size is deregulated. GMR-GAL4-driven expression of human $R P L 8^{D N}$ causes a small eye phenotype. These eyes, however, contain a normal number of ommatidia. Similarly, when human $R P L 8^{D N}$ is expressed in the wing, the observed cell size reduction contributes $\sim 84 \%$ to the overall decrease in the size of the wing, while a reduction in cell number contributes only $\sim 16 \%$ to the decrease in wing size. Therefore, the loss of Drosophila $R P L 8$ activity affects organ size mostly by deregulating cell size.

We have shown that Drosophila RPL8 is a potent regulator of cell and organ size. Recent studies have shown that alterations of many components in the insulin pathway also affect the same properties [32]. For example, inactivation of $T s c 1$, a negative regulator of the insulin pathway, or overexpression of Drosophila InR, a positive regulator of the insulin pathway, leads to an increase of both cell size and organ size $[40,41]$. On the other hand, inactivation of positive components of the insulin pathway results in an opposite phenotype [40]. The Drosophila S6K gene has been previously shown to act as a downstream component in the insulin pathway in the cell size control [33]. Inactivation of Drosophila $S 6 K$ causes the smaller cell size phenotype akin to the effect caused by the loss of Drosophila RPL8 activity. The human $R P L 8^{D N}$ and Drosophila RPL ${ }^{R N A i}$ phenotypes strongly suggest that RPL8 might play a role in insulin signaling. Indeed, we show that phenotypes associated with increased insulin signaling can be suppressed by human $R P L 8^{D N}$ and Drosophila RPL $8^{R N A i}$. We also show that Drosophila RPL8 genetically interacts with Drosophila Myc in regulating growth. Together, our results point to the critical role of Drosophila RPL8 in multiple cell growth signaling pathway and in the regulation of cell and tissue size.

\section{Materials and Methods}

\footnotetext{
Cloning of full-length human cDNAs into P-element transformation vectors

The $p U A S T$ vector contains a UAS promoter for gene expression and flanking P-element terminal repeats for integration into the genome. The presence of the mini-white ${ }^{+}$marker gene allows the selection of transformants with red eyes. $p U M A$ is a modified vector for $p U A S T$ containing a modified multiple cloning sequence with the restriction sites PI-Sce I and I-Ceu I (Supplementary information, Figure S2). This enables the efficient cloning of fulllength human cDNAs derived from a human kidney cDNA library made in the $p O T B 7$ vector.
} 
Fly strains and P-element transformation

Flies are maintained on a standard corn-agar medium at $25{ }^{\circ} \mathrm{C}$. P-element constructs are injected into $w^{1118}$ embryos as described previously. To screen for germline integration, the enclosed flies are backcrossed into $w^{1118}$ flies, where the presence of red-eyed progenies indicates successful transformation. We used $y w ; A d v /$ $\mathrm{CyO} ; \mathrm{Sb} / \mathrm{TM} 6 \mathrm{~B}$ flies to cross to individual transgenic lines to balance and map the transgenes. For each human gene construct, at least three independent transgenic lines are used to assay for overexpression phenotypes. A panel of eight different GAL4 lines is chosen since their expression patterns are in tissues that would give easily scorable and identifiable phenotypes. These lines include actin-GAL4, elav-GAL4, en-GAL4, ey-GAL4, GMR-GAL4, pnr-GAL4,ptc-GAL4, and $v g-G A L 4$. We also generated additional transgenic lines for overexpression of genes that we are interested in for pursuing further characterization. These include $U A S$ p27 $7^{\text {S10A }}, U A S-d x 16, U A S-D r o s o p h i l a ~ R P L 8$, and UAS-Drosophila $R P L 8^{R N A i}$. Additional details of the genotypes are described in the figure legends.

\section{Microscopy and immunohistochemistry}

The line $y w, h s F L P ; A c t i n>y^{+}>$Gal4, UAS-GFP was used to make "FLIP-out" clones in the anterior wing margin [22]. Clones were induced in 72-h-old larvae by a single 1-h heat shock at 37 ${ }^{\circ} \mathrm{C}$. This procedure induces recombination between the FRT sites of Actin $>y^{+}>$Gal4, which, consequently, removes the $y^{+}$cassette within the clones, thus allowing gene expression under the control of Actin-Gal4. Adult wings were dissected in 75\% ethanol, mounted in DPX Mounting Medium (Electron Microscopy Sciences) and examined using light microscopy.

Scanning electron microscopy (SEM) was performed as described [42]. All SEM images from at least three female adult eyes of each genotype were analyzed. The areas of all intact ommatidia were measured using the histogram function of the Adobe Photoshop software to determine the ommatidia size. The wild-type size was set arbitrarily as 1 , to which all other values were normalized.

Eye discs of third instar larvae were dissected, fixed, stained, and mounted as described [43]. To quantify the number of cells undergoing apoptosis, acridine orange staining was performed according to Neufeld et al. [44]. The following antibodies and dilutions were used: FITC-conjugated goat anti-GFP (1:200; Santa Cruz), mouse anti-ELAV (1:500; DSHB), and TRITC-conjugated goat anti-mouse (1:1 000; Santa Cruz).

\section{Acknowledgments}

We are grateful to Xizhi Ma, Junnian Zhou, Tianhong $\mathrm{Xu}, \mathrm{Xu}$ Liu, Xu Ding, Yang Liu, Ying Peng, Congwu Chi, Yiying Shang, Mingyao Ying, Sheng Ding, Lei Sun, Lei Tian, Huanhu Zhu, Hua Huang, Hongmei Li, and Xiaomo $\mathrm{Wu}$ for cDNA constructs and partial transgenic work, and Lihui Zhou (East China University of Science and Technology, China) for scanning electron microscopy. We thank Duc Nguyen (Yale University, USA) for critical reading and editing of this manuscript. This work is supported by grants from the National Natural Science Foundation of China (Grant Nos. 30030080, 39970408 and 30470840), National Basic Research Program of China (973) (Grant No. 2006CB806700).

\section{References}

1 Benson DA, Karsch-Mizrachi I, Lipman DJ, Ostell J, Wheeler DL. GenBank. Nucleic Acids Res 2006; 34:D16-D20.

2 Venter JC, Adams MD, Myers EW, et al. The sequence of the human genome. Science 2001; 291:1304-1351.

3 Lander ES, Linton LM, Birren B, et al. Initial sequencing and analysis of the human genome. Nature 2001; 409:860-921.

4 Collins FS, Green ED, Guttmacher AE, Guyer MS. A vision for the future of genomics research. Nature 2003; 422:835847.

5 Adams MD, Celniker SE, Holt RA, et al. The genome sequence of Drosophila melanogaster. Science 2000; 287:21852195.

6 Phelps CB, Brand AH. Ectopic gene expression in Drosophila using GAL4 system. Methods 1998; 14: 367-379.

7 Rørth P. A modular misexpression screen in Drosophila detecting tissue-specific phenotypes. Proc Natl Acad Sci USA 1996; 93:12418-12422.

8 Brand AH, Perrimon N. Targeted gene expression as a means of altering cell fates and generating dominant phenotypes. Development $1993 ; \mathbf{1 1 8}: 401-415$.

9 Crosby MA, Goodman JL, Strelets VB, Zhang P, Gelbart WM, FlyBase Consortium. FlyBase: genomes by the dozen. Nucleic Acids Res 2007; 35:D486-D491.

10 Freeman M. Reiterative use of the EGF receptor triggers differentiation of all cell types in the Drosophila eye. Cell 1996; 87:651-660.

11 Quiring R, Walldorf U, Kloter U, Gehring WJ. Homology of the eyeless gene of Drosophila to the Small eye gene in mice and Aniridia in humans. Science 1994; 265:785-789.

12 Huang AM, Rubin GM. A misexpression screen identifies genes that can modulate RAS1 pathway signaling in Drosophila melanogaster. Genetics 2000; 156:1219-1230.

13 Paumard-Rigal S, Zider A, Vaudin P, Silber J. Specific interactions between vestigial and scalloped are required to promote wing tissue proliferation in Drosophila melanogaster. Dev Genes Evol 1998; 208:440-446.

14 Fitzpatrick CA, Sharkov NV, Ramsay G, Katzen AL. Drosophila myb exerts opposing effects on $\mathrm{S}$ phase, promoting proliferation and suppressing endoreduplication. Development 2002; 129:4497-4507.

15 Hinz U, Giebel B, Campos-Ortega J. The basic-helix-loophelix domain of Drosophila lethal of scute protein is sufficient for proneural function and activates neurogenic genes. Cell 1994; 76:77-87.

16 Calleja M, Moreno E, Pelaz S, Morata G. Visualization of gene expression in living adult Drosophila. Science 1996; 274:252255.

17 Cheng T, Rodrigues N, Dombkowski D, Stier S, Scadden DT. Stem cell repopulation efficiency but not pool size is governed by p27kip1. Nat Med 2000; 6:1235-1240.

18 Malek NP, Sundberg H, McGrew S, Nakayama K, Kyriakides TR, Roberts JM. A mouse knock-in model exposes sequential proteolytic pathways that regulate $\mathrm{p} 27 \mathrm{Kip} 1$ in G1 and S phase. Nature 2001; 413:323-327. 
19 Oh S, Kim TK, Hwang DS, Yim J. Involvement of retinoblastoma protein in p27Kip1-induced apoptosis. Cancer Lett 2000; 148:105-110.

$20 \mathrm{Kim}$ WH, Ghil KC, Lee JH, et al. Involvement of p27kip1 in ceramide-mediated apoptosis in HL-60 cells. Cancer Lett 2000; 151:39-48.

21 Toyoshima H, Hunter T. p27, a novel inhibitor of G1 cyclinCDK protein kinase 14 activity, is related to p21. Cell 1994; 78:67-74.

22 Ito K, Awano W, Suzuki K, Hiromi Y, Yamamoto D. The Drosophila mushroom body is a quadruple structure of clonal units each of which contains a virtually identical set of neurones and glial cells. Development 1997; 124:761-771.

23 Franke KH, Miklosi M, Goebell P, et al. Cyclin-dependent kinasex inhibitor p27kip1 is expressed preferentially in early stages of urothelial carcinoma. Urology 2000; 56:689-695.

24 Koff A. How to decrease p $27^{\text {Kip } 1}$ levels during tumor development. Cancer Cell 2006; 9:75-76.

25 Ishida N, Kitagawa M, Hatakeyama S, Nakayama K. Phosphorylation at serine 10 , a major phosphorylation site of p27Kip1, increases its protein stability. J Biol Chem 2000; 275:25146-25154.

26 Maniatis T, Tasic B. Alternative pre-mRNA splicing and proteome expansion in metazoans. Nature 2002; 418:236-243.

27 Jumaa H, Wei G, Nielsen PJ. Blastocyst formation is blocked in mouse embryos lacking the splicing factor SRp20. Curr Biol 1999; 9:899-902.

28 O'Neill EM, Rebay I, Tijan R, Rubin GM. The activities of two Ets-related transcription factors required for Drosophila eye development are modulated by the Ras/MAPK pathway. Cell 1994; 78:137-147.

29 Huang H, Potter CJ, Tao W, et al. PTEN affects cell size, cell proliferation and apoptosis during Drosophila eye development. Development 1999; 126:5365-5372.

30 Ruhf ML, Meister M. The Drosophila homologue of ribosomal protein L8. Insect Biochem Mol Biol 1999; 29:349-353.

31 Potter CJ, Pedraza T, Xu T. Akt regulates growth by directly phosphorylating Tsc2. Nat Cell Biol 2002; 4:658-665.
32 Stocker H, Hafen E. Genetic control of cell size. Curr Opin Genet Dev 2000; 10:529-535.

33 Montagne J, Stewart MJ, Stocker H, Hafen E, Kozma SC. Drosophila S6 kinase: a regulator of cell size. Science 1999; 285:2126-2129.

34 Stowers RS, Schwarz TL. A genetic method for generating Drosophila eyes composed exclusively of mitotic clones of a single genotype. Genetics 1999; 152:1631-1639.

35 Laura AJ, David AP, Bruce AE, Robert NE, Peter G. Drosophila myc regulates cellular growth during development. Cell 1999; 9:779-790.

36 Abdelilah-Seyfried S, Chan YM, Zeng C, et al. A gain-offunction screen for genes that affect the development of the Drosophila adult external sensory organ. Genetics 2000; 155:733-752.

37 Kraut R, Menon K, Zinn K. A gain-of-function screen for genes controlling motor axon guidance and synaptogenesis in Drosophila. Curr Biol 2001; 11:417-430.

38 Rørth P, Szabo K, Bailey A, et al. Systematic gain-of-function genetics in Drosophila. Development 1998; 125:1049-1057.

39 Potter CJ, Pedraza LG, Huang H, Xu T. The tuberous sclerosis complex (TSC) pathway and mechanism of size control. Biochem Soc Trans 2003; 31:584-586.

40 Potter CJ, Xu T. Mechanisms of size control. Curr Opin Genet Dev 2001; 11:279-286.

41 Brogiolo W, Stocker H, Ikeya T, Rintelen F, Fernandez R, Hafen E. An evolutionarily conserved function of the Drosophila insulin receptor and insulin-like peptides in growth control. Curr Biol 2001; 11:213-221.

42 Potter CJ, Huang H, Xu T. Drosophila Tsc1 functions with Tsc2 to antagonize insulin signaling in regulating cell growth, cell proliferation, and organ size. Cell 2001; 105:357-368.

$43 \mathrm{Xu} \mathrm{T}$, Rubin GM. Analysis of genetic mosaics in developing and adult Drosophila tissues. Development 1993; 117:12231237.

44 Neufeld TP, de la Cruz AF, Johnston LA, Edgar BA. Coordination of growth and cell division in the Drosophila wing. Cell 1998; 93:1183-1193.

(Supplementary information is linked to the online version of the paper on the Cell Research website.) 